\title{
Compliance of people with diabetes in the National Diet and Nutrition Survey (2008-2016) to the National Institute for Health and Care Excellence dietary guidelines for type 2 diabetes management
}

\author{
H.A. Hussain ${ }^{1}$, Y. Jin ${ }^{1}$, M. Gongora ${ }^{2}$ and L.J. Dunford ${ }^{1}$ \\ ${ }^{1}$ Faculty of Health and Life Sciences, De Montfort University, Leicester, UK and \\ ${ }^{2}$ Faculty of Computing, Engineering and Media, De Montfort University, Leicester, UK
}

Type 2 Diabetes Mellitus (T2DM) is an endocrine disorder in which the post-prandial release of insulin is unable to sufficiently control blood glucose levels, resulting in hyperglycaemia. T2DM has a strong association with lifestyle factors, such as diet, exercise and socioeconomic status. Age and ethnicity are also risk factors ${ }^{(1)}$. The current dietary guidelines published by the National Institute for Health and Care Excellence (NICE) for managing T2DM include an increased consumption of low glycaemic index sources of carbohydrate (e.g. fruit, vegetables, wholegrains and pulses), complex carbohydrates, oily fish and low-fat dairy products. Consumption of food rich in saturated and trans-fatty acids should be reduced ${ }^{(2)}$. However, evidence is lacking about whether people with diabetes comply with these guidelines. The aim of this study is to assess the compliance of people with diabetes from the National Diet and Nutrition Survey (NDNS) dataset to the NICE dietary guidelines for T2DM management.

NDNS Rolling Programme data for 2008 -2016 were retrieved online via the UK Data Service. Participants aged 18 years and older were included. Dietary data (means of 4-day dietary records) were sorted into three groups: Diabetic Known (self-reported diabetic participants, $\mathrm{n}=178$ ), Diabetic Unknown (participants identified as diabetic by a blood glucose level of $7 \mathrm{mmol} / \mathrm{L}$ or higher, or a glycohaemoglobin level of $6.5 \%$ or higher $\left.{ }^{(3,4)}, \mathrm{n}=80\right)$ and a control group without any indicators of diabetic status $(\mathrm{n}=1601)$. To assess dietary compliance, levels of sucrose, fibre and total fruit and vegetable intakes were considered. Data were analysed using SPSS v26 ANOVA and a multiple regression analysis with group, age, sex and equivalised household income included as the predictors. Values are expressed as means with $95 \%$ confidence intervals.

Sucrose was significantly lower $(\mathrm{P}<0.01)$ in the diabetic known group, $29.5(26.3-32.7)$ g/day compared with diabetic unknown, 41.4 (35.9-46.8) g/day and control group, $43.4(42.1-44.8) \mathrm{g} / \mathrm{day}$. As a percentage of total energy intake sucrose was significantly lower $(\mathrm{P}<0.001)$ in the diabetic known group $4.03(3.65-4.41) \%$, vs. $5.53(4.92-6.15) \%$ (diabetic unknown) and 5.61 $(5.47-5.74) \%$ (control). However, fibre, fruit and vegetable intake were not higher in the diabetic known group compared with the other groups. Multiple linear regression analysis showed that diabetic status along with age, sex and equivalised income were not strong predictors of the dietary outcomes.

The current analyses suggest that people diagnosed with diabetes did not comply with the NICE dietary guidelines for T2DM management, other than reducing sucrose intake. This highlights the need for effective interventions to improve compliance, and more personalised dietary guidelines rather than general information could be considered as an alternative.

\section{References}

1. National Institute for Health and Clinical Excellence. Appendix B: Scopes for NICE clinical guideline update 2015, NICE clinical guideline 87 \& NICE clinical guideline 66 (2015).

2. National Institute for Health and Care Excellence. Type 2 diabetes in adults: management (2015) [Available at: https://www.nice.org.uk/guidance/ ng28/chapter/1-Recommendations\#dietary-advice-and-bariatric-surgery].

3. Diabetes Digital Media Ltd. Blood Sugar Level Ranges (2019) [Available at: https://www.diabetes.co.uk/diabetes_care/blood-sugar-level-ranges. html].

4. National Institute of Diabetes and Digestive and Kidney Diseases. The A1c Test and Diabetes (2018) [Available at: https://www.niddk.nih.gov/ health-information/diagnostic-tests/a1c-test?dkrd=/health-information/diabetes/overview/tests-diagnosis/alc-test]. 